\title{
Children's Understanding of Food and lts Functions: A Preliminary Study of the Development of Concepts of Nutrition*
}

\author{
HenRy M. Wellman \\ University of Michigan \\ Ann Arbor, MI 48109 \\ CARL N. Johnson \\ University of Pittsburgh \\ Pittsburgh, PA
}

\begin{abstract}
Children's developing conceptions of how the body functions nutritionally were examined. Two different tasks provided converging findings. One task asked children to judge the causes of certain end states (e.g., why $X$ is fat). The second task asked children to judge the result of certain contrasting diets (e.g., twin-1 eats twice as much candy as twin-2). The results evidenced; (a) systematic misconceptions on the part of kindergarteners that were overcome by sixth grade; and (b) other fairly sophisticated conceptions present even at the youngest ages tested. The research provides a needed first step toward describing the naive theories of nutrition possessed by children at different ages. Knowledge of childrens' theories of nutrition are essential for optimal planning of curricula and methods in nutrition education.
\end{abstract}

The past decade has seen an upsurge of interest and research in nutrition education beginning with the 1969 White House Conference on Food, Nutrition and Health. For children the goal of nutrition education is to increase the understanding of the body's use of food, the nutritional value of various foods and diets, and the importance and function of good nutrition for growth and health. Nutrition education programs are typically designed to present basic information from nutrition science. This involves identifying essential nutrition knowledge and

*This research was supported in part by a grant to the first author from General Mills, Inc. We thank Cathy Noblic for her research assistance and Bill Fabricius for his help in data analysis. Send reprint requests to: H. M. Wellman, Center for Human Growth and Development, University of Michigan, Ann Arbor, MI 48109. 
imparting it to children-primarily the character and presence of various nutrients and the content of the four food groups. The nature of these programs is reflected in the pre- and posttests given to children to assess their nutrition knowledge. These tests essentially assess nutrition facts, such as "What are the primary food groups?", "What is a calorie?", "True or false, tomatoes and potatoes are good sources of vitamin C" (Dwyer, Feldman, \& Mayer, 1970).

Missing from such an approach is an understanding of the underlying concepts about the body, and its use of food, which children possess independent of exposure to nutrition instruction. There are two related points here. First, theories of adult cognition distinguish between technical and popular concepts or knowledge (Glass \& Holyoak, 1975). In regard to knowledge about nutrition, on the one hand there would be technical concepts derived from such disciplines as biology and nutrition (e.g., about vitamins, cholesterol, or saturated and unsaturated fats), on the other hand are concepts reflecting commonsensical observations and understanding (e.g., "The more you eat the fatter you'll get"). Support for such a distinction comes from research on people's health beliefs. From this literature it is clear that health beliefs are heavily influenced by sources other than "expert" knowledge (Blumhagen, 1980; Chrisman, 1977). Indeed, there are two other major sources: A person's own experiences or observations, and popular or lay conceptions. In the present case, children surely have personal experiences with their own body, its ingestion of food and certain consequences thereof. They also receive messages from parents and others which reflect popular conceptions ("Too much candy will make you fat"; "'This gives you go-power"). It is highly likely that even very young children construct their own conceptions and misconceptions of nutrition incorporating these observations and attributions.

The second point derives from the cognitive development literature which suggests that the acquisition of new information, for example about nutrition, does not occur in isolation but rather is assimilated to preexisting conceptions (Piaget, 1970). Research has shown that what is learned from new information is highly dependent on the child's current stage of understanding related information (Kuhn, 1972; Turiel, 1969), that ability to remember new information depends on the extent and organization of previously acquired information (Chi, 1978), and that new information is best assimilated if it is only moderately discrepant from previously held beliefs (Kuhn, 1972; Siegler, 1978). In short, children's naive concepts of nutrition are an important but unstudied topic. Children undoubtedly construct theories of how their body works nutritionally and such notions interact with new information provided by nutrition instruction. In the present research we have taken a first step toward investigating these developing concepts of nutrition.

What sorts of conceptions about nutrition might children develop? Consider, for example, the following beliefs. Within certain limits amount of food consumed is related to amount of bodily tissue: "The more you eat the more you weigh." Within other limits amount of food consumed seems related to energy levels: "Too little food makes you weak and drowsy, enough makes you active 
and energetic, too much makes you drowsy and sluggish." Or consider knowledge about quantity/variety tradeoffs- "You can't exist on just green beans, no matter how much you eat." Conceptions such as these stem from a naive or popular theory of nutrition. The present effort to investigate the development of this sort of knowledge of nutrition was based on a three component model of the body's nutritional conversion system. These components seem to logically underlie common sense observations like those above. There are inputs to the body; there are outputs or products of the body; and there is the mediating role of the body itself. Important inputs are food, water, and other nutrients. These can vary across time or across individuals in amount, timing, and in quality or variety. Important outputs are energy, weight, tissue growth and replacement, and certain physical excretions. The question of interest is, what does the child know about the relations between input and output, about the nutritional conversion system? While this developing knowledge can be influenced by both naive conceptions and technical information, our goal was not to test children's store of technical facts, but instead to reveal their overall conceptions in the sense outlined above.

Some indirect evidence about children's concepts of nutrition can be derived from studies of their concepts of internal bodily organs (Contento, 1981; Dillon, 1935; Gellert, 1962, Nagy, 1953). For example, Gellert found that a majority of children as young as 5 were aware that food goes to the stomach. However, research of this type is limited in two respects. First, in focusing on the contents of the body (where organs or inputs such as the stomach and food reside) it provides limited insight into children's conceptions of the functioning of the body, specifically its nutritional functioning. Thus, while we know many younger children think food goes to the stomach, we do not know what they think about its relation to growth or weight. Second, the body concept research is based on openended interviews. Such interviews can provide interesting leads but they fail to provide a systematic assessment of the specific elements of children's conception. The present research, therefore, focused on children's knowledge of nutritional functioning, and the method used was one of standardized judgment tasks, not open-ended interviews. In an unexplored area such as this, no one study can fully reveal children's conceptions. The present research, therefore, constitutes a preliminary investigation, demonstrating a fruitful method and providing initial information toward a more complete future developmental description of concepts of nutrition.

Finally, while we have introduced this study by speaking of nutrition education specifically, knowledge of children's nutrition conceptions has broader relevance. Children's conceptions here are part and parcel of their growing knowledge of health and illness more generally. Knowledge of how the body functions has two complementary aspects: (a) normal operation-including nutritional knowledge; and (b) problematic operation-including illness and injury. Nutrition is, further, an important determinant of illness. Thus an understanding of children's developing concepts of nutrition has relevance for the growing body of re- 
search on children's health beliefs, encompassing knowledge of illness, injury, bodily organs, and nutritional functioning.

\section{METHODS}

\section{Subjects}

Fifteen kindergarteners (mean age 6 years; 3 months), 15 third graders (mean 9; 4), and 15 sixth graders (mean 12;6), were tested; 8 girls and 7 boys at each age. All children were students at an elementary school in suburban Pittsburgh serving a predominantly while middle-class population.

\section{Tasks}

Two tasks were developed, focusing on different aspects of the nutritional system. First, a task was used to test children's ideas about what caused differences in the outputs of two characters, such as differences in weight, height, health, strength, and energy. Here, pictures were shown of two children who differed in one dimension such as weight, height, etc., and subjects were asked what made the characters different. A second task focused on children's ideas about the consequences of variations in certain nutritional inputs. Here children were presented with pictures of identical twins and asked to judge whether differing future diets would result in differences between the twins. The Difference and Twin tasks wer presented in counterbalanced orders. In each task the characters presented matched the sex of the child. In the following descriptions, the male version is used.

Difference tasks. The child was shown five drawings of pairs of children where the depicted children differed on a relevant dimension (e.g., one skinny, one fat). The child was asked what caused the children to be different. The five items were presented in random order.

1. Fat/Skinny: These two boys are Jimmy and Johnny. You can see that Jimmy is really skinny and Johnny is pretty fat. What do you think made them different? . . . Anything else? . . . (If the answer is not related to food:) Do you think that what they ate might have made the difference?

2. Peppy/Lazy: This is peppy Danny and this is lazy Dave. Danny is real peppy and active and he really has a lot of energy. Dave is so lazy that all he ever does is sit around and sleep a lot. What do you think it was that made them different? (Plus probes as above.) 
3. Tall/Short: Billy, as you can see is really tall, and Bobby is the shortest kid in the class. What do you think made them different? (Other probes, as above.)

4. Healthy/Sick: These two boys are Ronnie and Reggie. Ronnie is really healthy. He is so healthy he never missed a day of school. Poor Reggie is always getting sick. He catches everything there is to catch. What do you think made them different? (Other probes.)

5. Strong/Weak: Todd is really weak and he can hardly even lift anything. Tom is the strongest kid around. What do you think made them different? (Other probes.)

Twin Tasks. In the Twin tasks the child was told about two identical twins. Line drawings of two identical boys were presented, and the child was instructed that one of the twins was going to have a different diet from the other for the upcoming year. The child was asked; (a) what would happen to the twins, and (b) whether there would be any changes in the target twin, as compared to the other twin. The 8 different diets were: the target twin (1) would eat twice as many vegetables as his twin; (2) would eat only green beans; (3) would drink twice as much water; (4) would consume only water; (5) would eat twice as much of everything; (6) would consume only food, no drink; (7) would eat twice as much dessert; (8) would eat only candy. Diet items were presented in random order.

\section{RESULTS}

\section{Difference Tasks}

The responses to the Difference tasks are summarized in Table 1. The responses coded here are children's answers to the initial difference questions- what made the two boys different? (e.g., one fat and one skinny)-and responses to the probe-do you think what they ate may have made the difference?' Questions of-

'The probe questions about food specifically were included in order to get as much information as possible in this preliminary study. Two concerns can be raised, however, about this procedure and data. First, perhaps inclusion of the probe in the early items induced a response set or demand where children then focused on food in later responses. This seems unlikely, however, since responses on first items (before a set would be induced) provide an almost identical picture to that in Table 1 . In Table 1, across all items, kindergarteners, third-, and sixth-graders gave Food responses 69\%, 92\%, and $82 \%$ of the time respectively. On the first items alone, the corresponding figures were $67 \%, 100 \%$, and $97 \%$ for the three ages. A second concern, involves not the demand characteristics across items but the demand characteristics of the probe itself. Perhaps, in response to the probe, children merely said "yes" food was a factor, because it was suggested to them, not because of their conceptions. However, there appeared to be no bias to respond yes to this question. If probed with this question, on the average children answered yes $57 \%$ and no $43 \%$ of the time. This even distribution of responses was 


\section{TABLE 1}

Response to Initial Question on the Difference Tasks What made to two boys different?)

\begin{tabular}{|c|c|c|c|}
\hline & $K^{\prime}$ & $3^{7}$ & $6^{\prime}$ \\
\hline \multicolumn{4}{|l|}{ Fat/Skinny } \\
\hline Food Responses & 14 & 15 & 15 \\
\hline Quantity of Food & 10 & 15 & 15 \\
\hline Quality of Food & 1 & 4 & 4 \\
\hline $\begin{array}{l}\text { Specific Foods } \\
\text { Tall/Short }\end{array}$ & 4 & 1 & 2 \\
\hline Food Responses & 9 & 15 & 7 \\
\hline Quontity of Food & 5 & 8 & 1 \\
\hline Quality of Food & 3 & 2 & 1 \\
\hline Specific Foods & 0 & 4 & 0 \\
\hline Age & 6 & 1 & 0 \\
\hline Heredity & 0 & 3 & 12 \\
\hline \multicolumn{4}{|l|}{ Peppy/Lazy } \\
\hline Food Responses & 7 & 13 & 15 \\
\hline Quantity of Food & 0 & 2 & 2 \\
\hline Quality of Food & 4 & 3 & 11 \\
\hline Specific Foods & 2 & 4 & 3 \\
\hline Exercise & 4 & 3 & 0 \\
\hline Sleep & 2 & 2 & 6 \\
\hline \multicolumn{4}{|l|}{ Strong Weak } \\
\hline Food Responses & 10 & 13 & 15 \\
\hline Quantity of Food & 6 & 5 & 1 \\
\hline Quality of Food & 1 & 6 & 13 \\
\hline Specific Foods & 2 & 3 & 2 \\
\hline $\begin{array}{l}\text { Exercise } \\
\text { Healthy/Sick }\end{array}$ & 8 & 9 & 5 \\
\hline Food Responses & 12 & 13 & 14 \\
\hline Quantity of Food & 2 & 5 & 1 \\
\hline Quality of Food & 6 & 5 & 10 \\
\hline Specific Foods & 4 & 6 & 3 \\
\hline Exposure & & & \\
\hline
\end{tabular}

$N=15$ at each age, however, multiple categories of response from one subject are included so that totals may be greater than 15.

ten elicited multiple responses from a given child, Table 1 thus presents the total number of children providing each response.

Results of the Difference tasks provide two kinds of information. First, they indicate whether children in some way regarded food as a means for achieving the various endstates. The first row under every heading in Table 1-Food responses-shows the number of responses designating food as a factor determining the depicted differences. Children of all ages were aware of the re-

apparent at all ages; kindergarteners, for example (who might be presumed to be most susceptible to some implicit demand to say yes), gave only $48 \%$ yes, $52 \%$ no. Finally, this probe does not seem to have unduly affected responses since very similar conceptions were revealed in a completely different task, the Twins tasks. 
lation of food to weight and health: as shown in Table 1 Food responses to Fat/Skinny and Healthy/Sick were frequent at all ages, and responses did not significantly differ across the ages ( $\chi^{2}$ tests here and in what follows). However awareness of the role of nutrition increased with age on the Strong/Weak- $\chi^{2}(1)=10.04, p<.01$, comparing kindergartners with older children ${ }^{2}$-and Peppy/Lazy items- $\chi^{2}(1)=3.56, p<.07$. Food related responses significantly decreased after third grade on the Tall/Short item- $\chi^{2}(1)=5.53, p<.02$.

Second, looking closer at the specifics of children's answers to the Difference tasks reveals three distinct aspects of nutrition understanding. As Table 1 shows, the specific content of children's food responses focused on either the Quantity of food, Quality of food or Specific foods. Quantity responses mentioned the amount of food as the critical variable ("He eats more", "He eats too much"). Quality responses referred to specific nutritional aspects of the person's diet ("He eats too much fattening stuff", "He doesn't eat the kind of things his doctor says", "He needs more vitamins and nutrients", "He needs more vegetables and stuff that's good for you, not just sweets"). Specific food responses indicated the presence or absence of one specific food ("He should eat hot peppers to get peppier", "He doesn't drink much milk so he's short", "He should drink lots of coffee to get peppier"). Not all Food responses (first row in Table 1) could be classified into one of these three specific categories (e.g., "It's what he eats"). In addition to the categorization of food related responses any other response that was provided by $20 \%$ or more of the children at some age is also shown in Table 1 . Inter-rater reliability in coding responses into these various categories was .95.

Children's specific answers are best considered item by item. Consider first the two items dealing with differences in sheer size of the person, Fat/Skinny and Tall/Short. In these cases the predominant food related response for the younger children was Quantity of food (given by $65 \%$ of those kindergartners who gave a food related response). With development there was a significant age increase in Quantity responses for Hat/Skinny- $\chi^{2}(1)=8.13, p<.01$-and a signiticant decrease for Tall/Short- $\chi^{2}(1)=4.68, p<.05$. Thus, for younger children any change in size (weight and height) was more likely to be seen as linked to changes in quantity of food. With age, understanding became more discriminate; changes in weight were seen as closely relating to quantities of food eaten, but changes in height came to be seen as predominantly responsive to other factors.

Consider next those two items concerned not with obvious size but with activity, either in the form of energy (Peppy/Lazy) or strength (Strong/Weak). There was an age increase in food related responses in general as reported above. In addition, there was an even sharper age increase in Quality of food responsesfor Peppy/Lazy, $\chi^{2}(1)=8.44, p<.01$; for Strong/Weak, $\chi^{2}(1)=11.75$,

${ }^{2}$ In all $\chi^{2}$ analyses where expected frequencies were small, age changes were tested by collapsing the two most similar yet adjacent age groups and comparing these to the remaining group. 
$p<.01$. Notice in contrast to Fat/Skinny and Tall/Short, Quantity was never the modal reply at any age. Where activity and not size was concerned, younger children were less likely to implicate a role for sheer amount of nutrition. Further, note in Table 1 that on these items exercise was often seen as particularly relevant. Activity was the basis of the difference described to the child (sitting still, lifting objects) and, especially for young children, activity (exercise) was often pointed to as the causal difference.

Children's responses to the Healthy/Sick item revealed that the vast majority of children at all ages knew health to be food related. This knowledge rested on Quality of food and Specific food responses. Essentially in both cases children identified a food (Specific food: "orange juice") or a general class of food (Quality of food: "vitamins", "what your doctor says to eat") that aided health.

In sum, younger children's understanding of the body seemed tied to obvious relationships between salient variables. Increases in activity (more exercise) were linked to increases in activity (more pep); increases in amount (more food) directly led to increases in amount (more weight or height). On the other hand, while every sixth grader replied that quantity of food eaten differentiated the fat from the skinny child, the prime factor determining height, for these older subjects, was not food at all. Instead, $80 \%$ of the sixth graders realized that Heredity ("born to be tall", "genes") accounts for most variation in height. For older children the quality of what you eat (as opposed to quantity) was the crucial factor for maintaining health (67\% of subjects), for increasing strength $(87 \%)$ and in accounting for energy levels ( $73 \%$ ). While older subjects were clearly more knowledgeable in their concepts of the nutritional system, the youngest children were not completely indiscriminate. For example, at all ages Quantity was a more frequent response for Fat/Skinny than for Tall/Short. And substantial numbers of kindergarteners and third graders said that Age ("He'll just have to get older') or Heredity were the key factors for Tall/Short.

\section{Twin Tasks}

The Twin tasks were analyzed with two goals in mind: (a) to determine if the trends and patterns discovered on the Difference tasks would be evident with a very different task; and (b) to extract additional information concerning children's notions of the consequences of varying sorts of inputs to the nutritional system. The Twin tasks were designed to include two types of inputs. In some cases one twin consumed twice as much of some input and in the other cases one twin consumed only one input. Results on the "double" items are presented in Table 2 and will be considered first; responses to the "only" items are considered second.

Double items. In the Difference tasks younger subjects seemed to overcorrelate quantity consumed and size. If this same tendency was apparent on the 
TABLE 2

Responses on the Twin Tasks to the Situations Depicting Doubled Amounts'

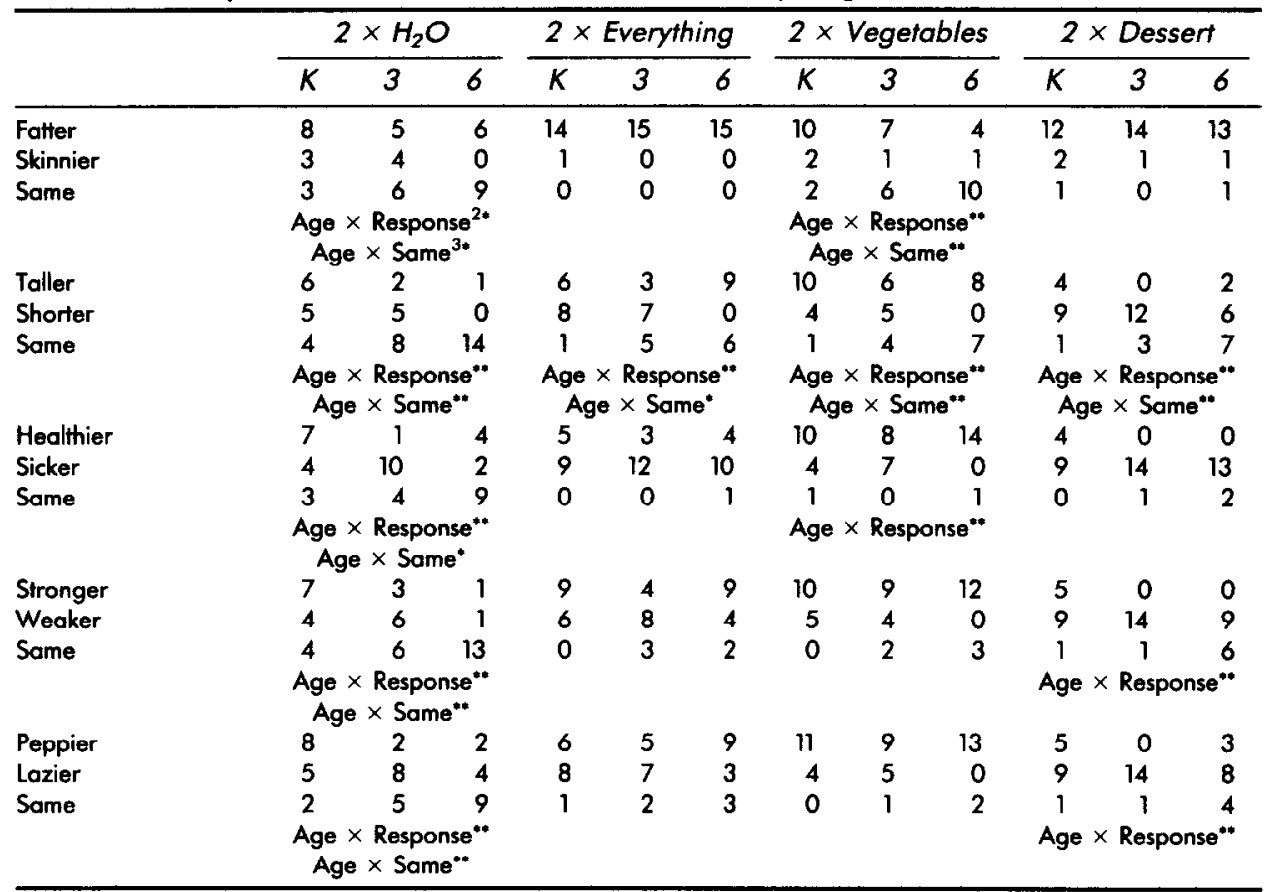

'Sometimes children gove no response thus totals are not always 15 at each age. At most only 2 children gave no response for any item.

${ }^{2}$ Significance levels are based on $3 \times 3$ chi-squares, except where frequencies were too low. In other cases $3 \times 2$ chi-squares were calculated where the two response categories with the smallest frequencies were collapsed. All significant results are shown.

${ }^{3}$ Significance levels are based on $3 \times 2$ chi-squares comparing Same responses to all others at each age.

$*_{p}<.10$

$* * p<.05$ or better

Twin tasks then two different patterns would be expected in Table 2. First, younger children could be expected to believe that relatively short-term changes in inputs would affect height as well as wcight. Thus, onc cxpected pattcrn would be the presence of age increases in the number of children responding "same" to the Taller/Shorter questions in Table 2: older children being more likely than younger children to know that doubled consumption would not influence height. As a look at row 2 of Table 2 shows, on all 4 items there were age changes in responses to the Taller/Shorter questions. In 3 of 4 cases age increases in Same responses were significant, and, in the remaining case it was marginally significant. 
Second, it may be expected that younger children would indiscriminately believe that increased consumption of anything would result in increases in size. Specifically, in Table 2 younger children could judge that all situations indicating doubled amount would lead to increased weight. Older children should be more likely to say $2 \times$ Everything and probably $2 \times$ Dessert would lead to increases in weight, but that with $2 \times \mathrm{H}_{2} \mathrm{O}$ and probably $2 \times$ Vegetables weight would not change. As Table 2 shows (row 1) there was a significant age related change of response on the $2 \times$ Vegetables item and a similar marginally significant pattern on $2 \times \mathrm{H}_{2} \mathrm{O}$. In both cases, the age differences were predominantly accounted for, as expected, by age increases in children's saying Same to these items. Thus older children were more likely to recognize that increases in amount of some inputs would not affect weight. In sum, as in the Difference tasks, younger children were more likely than older children to think that changes in amount consumed were directly and indiscriminately linked to changes in size, both height and weight.

Two other patterns of response in Table 2 are informative. Related to the above tendency for younger children to believe increased consumption of even water to be related to increased weight, young children also tended to think that increased water intake was related to many nutrition-related differences between the twins. Thus, they tended to say that the twin who drank twice as much water would become fatter, taller, healthier, stronger, and peppier. As Table 2 shows there were consistent age changes in these responses. Specifically, sixth graders were significantly more likely than younger children to say that extra water consumption would be unrelated (Same responses) to strength, energy, health, etc. Thus, sixth graders appeared to make a clear distinction between the role of water versus food, as nutritional inputs; younger children over-equated the two as similar "inputs."

The remaining pattern in a distinction between "good" foods and "bad" foods. Specifically at every age the predominant response to the $2 \times$ Vegetables item was that the twin eating more vegetables would be healthier, stronger, and peppier. On the other hand at every age the twin eating more dessert was judged to become sicker, weaker, and lazier. This pattern was even somewhat apparent on the Taller/Shorter question, though here (as discussed above) the sixth graders predominant response was that the twins would be the same. Comparing across items, 23 children gave a mixed response (said healthier for one item and sicker for the other) to the Healthy/Sick question for the vegetables vs. dessert items. Of these, 22 children said vegetables would make you healthier and dessert sicker, McNemar's $\chi^{2}=17.37, p<.001$. Of 24 mixed responders, 23 said vegetables would make you stronger, dessert weaker; McNemar's $\chi^{2}=18.38, p<.001$. Of 25 mixed responders all said vegetables would make you peppier, dessert lazier; McNemar's $\chi^{2}=23.04, p<.001$. This pattern was identical at all the ages.

This finding is related to the Specific food and Quality of food responses from the Difference tasks; children know some foods to be better for you, some 
worse. This is also apparent from other studies, where children have been asked what foods are in a good vs. a bad meal (e.g., Litman, Cooney, \& Stief, 1964). Indeed vegetables (or green beans) and dessert (or candy) were both included in the Twin tasks in order to control for responses based on these valences of certain foods.

Only items. The other half of the Twin tasks were meant to test the limits of children's understanding of certain nutritional relations. In these items, the child was asked what differences would occur if a person ate only one thing. Responses here were clear and consistent across all items. The vast majority of children at all ages realized that eating only one input was not good for you. Considered across the four items (Only $\mathrm{H}_{2} \mathrm{O}$, Only Food, Only Green Beans, Only Candy), 79\% of all responses indicated that the target twin would be shorter, $92 \%$ that he would be sicker, $93 \%$ that he would be weaker, and $90 \%$ that he would be lazier. In addition if he consumed only water or only food, $73 \%$ of all subjects judged the target twin would be skinnier, and if he ate only candy $78 \%$ judged the target would be fatter. In all of these items and for all of these judgments there were no age differences. For example, among kindergartners $72 \%$ of all responses across items indicated that the target twin would be shorter, $83 \%$ that he would be sicker, $75 \%$ that he would be weaker, and $83 \%$ that he would be lazier.

The singlc age related change in response to the "only" items concerned the Fatter/Skinnier question for the Only Green Beans item. Here children exhibited an increasing belief with age that a diet of this vegetable alone would lead to weight loss $-33 \%, 53 \%$ and $80 \%$ of the kindergartners through sixth graders predicting weight loss; $\chi^{2}(2)=6.66, p<.01$. Older children were aware that vegetables do not contribute as much to weight as calorie rich foods like meat and desserts. This is consistent with the data in Table 2 showing older children's awareness that even doubling one's diet of vegetables would not result in weight gain.

In short, all ages understood that a diet of one input alone has serious negative consequences. Indeed, a substantial minority of children noted that the target twin was likely to die if his only input was water $(20 \%, 20 \%$ and $33 \%$ of the kindergarteners through sixth graders) or if his only input was food with no water $(0 \%, 13 \%$, and $27 \%$ respectively).

\section{DISCUSSION}

Children's developing awareness of the nutritional functioning of the body was clear from their responses on these two tasks. Specifically, there were; (a) indications of systematic misconceptions on the part of kindergarteners that were overcome by sixth grade; yet (b) indications of certain discriminate conceptions present at even the youngest ages. 
Kindergarteners and to some extent third graders over-correlated changes of amount consumed with changes in size; for example treating height and weight as similarly influenced by amount eaten. They also tended to narrowly believe that changes in activity potentials (strength, energy) were derived from changes in activity itself (exercise) and not from less salient variables like nutrition. While younger children differentiated between some inputs which are "good" and "bad" for you they also tended to treat all inputs to the body as similar, in particular food and water were seen as having equivalent nutritional consequences. In the aggregate these results suggest that the young child possesses, at best, an oversimplified understanding of the body's use of nutrition. Especially, certain salient surface similarities color their thinking — quantity-size relations, activity-activity similarities and an over-equivalence of any inputs (food-water) and outputs (weight-height).

While younger children's knowledge of the body is marked by deficiencies when compared to older children, this is not the whole story. Even kindergarteners evidenced the beginning understanding of a number of important aspects of nutrition. The accuracies and inaccuracies of the young child can be summarized as follows. Knowledge about nutrition, as tested in this study, can be thought of as composed of three related accomplishments; (a) knowledge of a variety of relevant nutritional inputs and outputs; (b) knowledge that the inputs are functionally related to the outputs; and (c) knowledge of how inputs relate to outputs, i.e., knowledge of nutritional relationships and processes. Younger school age children seem well grounded, if still imperfect, in relation to $a$ and $b$. In regard to $a$ they know that there are different foods, especially there are good foods vs. bad foods. They know that food is not the only relevant factor (e.g., there is also exercise and age). And they know that there are different outcomes; though this understanding seems to distinguish only variations in size, activity, and health. In regard to $b$, younger children clearly know that inputs influence outputs. Their answers show that change in diet (especially eating more) is seen as relating to different outcomes (health, strength, weight, etc.) and they have knowledge of cetain crucial limits (e.g., just drinking water leads to death, eating only one thing even one good food is insufficient). The biggest change from kindergarten to sixth grade in this study seems to be in regard to $c$, understanding of how inputs relate to outputs. It is here that younger children's limitations are most obvious; thinking that food and water have equivalent nutritional consequences, that height and weight are equally products of different amounts of input, that activities but not nutrition are related to differences in energy and strength. Thus, what distinguishes the understanding of older children is their ability to construct a more integrated model of how different nutritional factors interact with each other and with various other factors in the human physiological system. This involves specifically the development of knowledge about an invisible system, or inferred set of relationships which go beyond surface or apparent similarities.

The goal of developmental description is to chart the progression of states, behaviors, or knowledge that characterize children as they grow older. Accurate 
developmental descriptions are of considerable practical utility. Specifically, the development of effective curricula requires information about children's normally advancing knowledge in the relevant domain: effective instruction involves presenting new information in terms of present conceptions and understanding; educational objectives must consider age-related sources of miscomprehension, and stages or styles of thinking which constrain acquisition of presented information. Given a need for developmental descriptions in the realm of nutrition, why not simply infer probable conceptions from general developmental theories such as Piaget's (see Contento, 1981)? For example, one might predict that 6- and 7-yearolds would not be able to understand the four primary food groups, given the general Piagetian description of the acquisition of classification skills. The trouble is that these general schemes are notoriously imprecise when it comes to predicting knowledge development in a specific content domain. "Our experience has been that we cannot predict how the forms of cognition outlined by Piaget and Werner will be manifested in a particular content area" (Bribace \& Walsh, 1979, p. 287). For example, the knowledge that most kindergarteners are in the preoperational stage of cognitive development would not allow one to predict that they understand that a diet of one input is unhealthy, but also misbelieve that increased consumption of water will lead to long-term changes in height. Children's naive conceptions about nutrition must themselves be investigated.

The present research represents only a beginning in this process, sampling a small number of subjects from a limited age-range and on only a subset of possible nutrition-related conceptions. However, the current demonstration has important strengths. Most important was the use of two converging, structured judgment tasks. Unlike open-ended interviews structured judgment tasks focus more specifically on a set of conceptual relations, and are less reliant on a child's abilities at spontaneous verbal expression. Further, the primary conclusions were replicated across two different tasks. The current methods could be usefully extended to other nutrition concepts, in future research, to provide a full developmental description of nutrition-related conceptions. The potential utility of a developmental description for this conceptual domain is also apparent from the present preliminary findings. Such a description can reveal things that younger children do not know, or misunderstand, and which are therefore worthy of instruction. In addition, it can reveal concepts which young children already possess, or partly understand, that therefore can be exploited to aid instruction, by basing presentation of new information upon old meaningful knowledge.

\section{REFERENCES}

Bibace, R., \& Walsh, M. E. Developmental stages in children's conception of illness. In G. Stone, F. Cohen \& N. Adler (Eds.), Health Psychology: A Handbook. San Francisco: Jossey-Bass, 1979. Blumhagen, D. Hypertension: A folk illness with a medical name. Culture, Medicine and Psychiatry, $1980,4,197-227$ 
Chi, M. T. H. Knowledge structures and memory development. In R. S. Siegler (Ed.), Children's Thinking: What develops? Hillsdale, NJ: Lawrence Erlbaum Associates, 1978.

Chrisman, N. The health seeking process: An approach to the natural history of illness. Culture, Medicine and Psychiatry, 1977, 1, 351-378.

Contento, I. Children's thinking about food and eating-A Piagetian-based study. Journal of Nutrition Education, 1981, 13, 586-590.

Dillon, M. S. Attitudes of children toward their own bodies and those of other children. Child Development, 1935, 5, 165-176.

Dwyer, J. T., Feldman, J. J., \& Mayer, J. Nutritional literacy of high school students. Journal of Nutrition Education, 1970, 2, 59-66.

Geliert, E. Children's conception of the content and functions of the human body. Genetic Psychology Monographs, 1962, 65, 293-401.

Glass, A. L., \& Holyoak, K. J. Alternative conceptions of semantic memory. Cognition, 1975, 3, 313-339.

Kuhn, D. Mechanisms of change in the development of cognitive structures. Child Development, $1972,43,833-844$.

Litman, T. J., Cooney, J. P., \& Stief, R. The views of Minnesota school children on food. Journal of the American Dietetic Association, 1964, 45, 433-440.

Nagy, M. H. Children's concepts of some bodily functions. Journal of Genetic Psychology, 1953, 22, 359-378.

Piaget, J. Piaget's theory. In P. Mussen (Ed.), Carmichael's manual of child psychology (Vol. 1). New York: John Wiley \& Sons, 1970.

Siegler, R. S. The origins of scientific reasoning. In R. S. Siegler (Ed.), Children's Thinking: What develops? Hillsdale, NJ: Lawrence Erlbaum Associates, 1978.

Turiel, E. Developmental processes in the child's moral thinking. In P. Mussen, J. Langer, \& M. Covington (Eds.), Trends and issues in developmental psychology. New York: Holt, Rinehart \& Winston, 1969.

White House Conference on Food, Nutrition, and Health. Recommendations of panels on nutrition education and teaching. Journal of Nutrition Education, 1969, 1, 24-39. 